\title{
Vibrations Analysis of Cracked Nanobeams Using Quadrature Technique
}

\author{
Mohamed Abd Elkhalek*, Tharwat Osman, Mohamed Saad Matbuly \\ Department of Engineering Mathematics and Physics, Faculty of Engineering, Zagazig University, Zagazig, Egypt \\ Email address: \\ mohamed_abdelkhalek4444@yahoo.com(M. A. Elkhalek) \\ ${ }^{*}$ Corresponding author

\section{To cite this article:} \\ Mohamed Abd Elkhalek, Tharwat Osman, Mohamed Saad Matbuly. Vibrations Analysis of Cracked Nanobeams Using Quadrature \\ Technique. Engineering Mathematics. Vol. 3, No. 1, 2019, pp. 19-29. doi: 10.11648/j.engmath.20190301.15
}

Received: June 1, 2019; Accepted: July 8, 2019; Published: July 17, 2019

\begin{abstract}
This work concerns with free vibration analysis of cracked nanobeam problems. Based on Eringen's nonlocal elasticity theory, the governing equation of Euler-Bernoulli and Timoshenko nanobeams, are derived. It is assumed that strain at a certain point is a function of the strains at all points within the influence domain. The cracked beam is modeled as multisegments connected by a rotational spring located at the cracked sections. This model promotes discontinuities in rotational displacement due to bending which is proportional to bending moment transmitted by the cracked section. Polynomial based differential quadrature method is employed to solve the problem. Derivatives of the field quantities are approximated as a weighted linear sum of the nodal values. For different supporting cases, the boundary conditions are directly substituted in the equation of motion, such that the problem is reduced to that of linear homogeneous algebraic system. This suggested numerical scheme accurately determined angular frequencies of the problem. A comparative study is tabulated to compare the obtained results with the previous ones. Further, a parametric study is introduced to investigate the influence of crack locations, crack severity and the nonlocal scale parameter on the obtained results. The obtained results recorded that frequency values decrease with the increasing of both of crack severity and the nonlocal scale parameter. The results of the proposed scheme may be applied for structural health monitoring.
\end{abstract}

Keywords: Cracked Nanobeam, Free Vibration, Euler-Bernoulli Theory, Timoshenko Theory, Differential Quadrature Method

\section{Introduction}

Nanostructures are commonly used because of their large Young's modulus, yield strength, flexibility, and conductivity properties. According to application of nanobeams in nanoelectromechanical systems and in biotechnology, it is important to investigate the vibrational behavior of nanobeams [1]. In nonlocal theory of elasticity, stress at a point is function of strains at all points in the continuum, while stress at a point is function of strain at the same point in local elasticity [2-6].

Several publications concerned with the analysis of noncracked nanobeam problems. Reddy [7] studied analytically vibration of nanobeams. Wang et al. [8] studied analytically vibration of nonlocal Timoshenko beams. Behera [9] studied free vibration of Euler and Timoshenko nanobeams using boundary characteristic orthogonal polynomials. In [10] the orthogonal collocation method is applied to study the free vibrations of nonlocal Timoshenko beams by using piecewise cubic Hermite polynomials. Eltaher [11] studied the vibration analysis of Euler-Bernoulli nanobeams using finite element method.

The crack can make the structures more flexible and so are able to decrease the natural frequencies of the structures [1215]. As a result, analysis of such structures may be applied for structural health monitoring. Also, several publications concerned with the analysis of cracked nanobeam problems. Loya [16] investigated free vibration of cracked EulerBernoulli nanobeams using nonlocal elasticity model. Sourki and Hoseini [17] investigated vibration of a cracked nanobeam based on the modified couple stress theory within the framework of Euler-Bernoulli beam theory. Also, they simulated a cracked nanobeam with the nonlocal modified couple stress theory and investigated the mechanical 
parameters with analytical method in [18]. Bahrami [19] used wave propagation methods to analyze vibration in multicracked Euler-Bernoulli nanobeams. Wang K [20] investigated the Timoshenko beam model for vibration analysis of a cracked nanobeam with surface energy. Torabi [21] considered analytically free vibration analysis of craked Timoshenko beam problem. Soltanpour [22] studied the vibration of functionally graded nanobeams resting on Winkler foundation using Timoshenko theory.

Although all these publications, analysis of cracked nanobeams still attractive. Recently, differential quadrature method is introduced as promising numerical technique. This method leads to very accurate results by using small number of nodal points [23-26]. Rotational spring model is employed to simulate the crack existence.

This work extends application of differential quadrature method for free vibration analysis of cracked nanobeams. The technique is applied for each of Euler-Bernoulli and Timoshenko beams with different boundary conditions. For different supporting cases, the angular frequency of the cracked nanobeam, is obtained. As well as, a parametric study is introduced to investigate the influence of crack locations, crack severities, and the nonlocal scale parameter on the obtained results.

\section{Formulation of the Problem}

Consider a nanobeam of length $\mathrm{L}$, containing multi-cracks located at a distance $d_{l}(l=1,2, \ldots, \mathrm{n})$ from the left end, as shown in Figure 1. The beam is divided into $(n+1)$ subbeams, while the cracks are modeled as a rotational springs at the crack positions. If we assume harmonic behavior of the problem, the field quantities can be expressed as:

$$
F(x, t)=f(x) e^{i \omega t}
$$

Where $f(x)$ denotes to field quantities, $\mathrm{x}$ is the longitudinal coordinate measured from the left end of the beam, $\mathrm{t}$ is time, $\omega$ is the natural frequency.

\subsection{Nonlocal Euler-Bernoulli Beam}

The governing equation based on nonlocal Euler-Bernoulli theory (EBT) for each sub-beam can be written as [16]:

$$
\frac{d^{4} w^{l}(x)}{d x^{4}}+\omega^{2}\left(e_{0}^{l} a^{l}\right)^{2} \frac{\rho^{l} A^{l}}{E^{l} I^{l}} \frac{d^{2} w^{l}(x)}{d x^{2}}-\omega^{2} \frac{\rho^{l} A^{l}}{E^{l} I^{l}} w^{l}(x)=0,(l=1, \mathrm{n}+1)
$$

Where $w^{l},(l=1, \mathrm{n}+1)$, is the deflection of sub-beam $l$, $\left(e_{0}^{l} a^{l}\right)^{2}$ is the scale coefficient that incorporates the small scale effect of sub-beam $l, E^{l}$ is Young's modulus of subbeam $l, I^{l}$ is the second moment of area of sub-beam $l, \rho^{l}$ is the mass density of sub-beam $l, A^{l}$ is the cross-sectional area of sub-beam $l$.

\subsection{Nonlocal Timoshenko Beam}

Also, the governing equations based on nonlocal Timoshenko beam theory (TBT) for each sub-beam can be written as [8]:

$$
\begin{array}{lr}
\qquad E^{l} I^{l} \frac{d^{2} \phi^{l}}{d x^{2}}-K_{s}^{l} G^{l} A^{l}\left(\phi^{l}+\frac{d w^{l}}{d x}\right)+\omega^{2} \rho^{l} I^{l} \phi^{l}-\left(e_{0}^{l} a^{l}\right)^{2}\left(\omega^{2} \rho^{l} A^{l} \frac{d w^{l}}{d x}+\omega^{2} \rho^{l} I^{l} \frac{d^{2} \phi^{l}}{d x^{2}}\right)=0, \\
\qquad K_{S}^{l} G^{l} A^{l}\left(\frac{d \phi^{l}}{d x}+\frac{d^{2} w^{l}}{d x^{2}}\right)+\omega^{2} \rho^{l} A^{l} w^{l}=0,(l=1, \mathrm{n}+1)(4) & \text { expressed as }[18,21],\left(\mathrm{x}=d_{l},(l=1, \mathrm{n})\right) \text { : } \\
\text { Where } G^{l} \text { is the shear modulus of sub-beam } l \text { and } \mathrm{K}_{s}^{l} \text { is the } & w^{l}=w^{l+1},(l=1, \mathrm{n}) \\
\begin{array}{l}
\text { Shear correction factor of sub-beam } l, \mathrm{~L} \text { is the length of the } \\
\text { whole beam. }
\end{array} & M^{l}=M^{l+1},(l=1, \mathrm{n}) \\
\begin{array}{l}
\text { We may take } \mathrm{K}_{S}^{l}=0.563 \text { for all sub-beams as given by }[8] . \\
\text { 2.3. Boundary Conditions }
\end{array} & Q^{l}=Q^{l+1},(l=1, \mathrm{n}) \\
\end{array}
$$

\subsection{Boundary Conditions}

The boundary conditions of the problem at external boundaries, $(\mathrm{x}=0$ and $\mathrm{x}=\mathrm{L})$ can be expressed as follows:

For a simply supported end:

$$
w^{l}=0 \text { and } M^{l}=0
$$

For a clamped supported end:

$$
w^{l}=0 \text { and } \Phi^{l}=0
$$

For a free supported end:

$$
Q^{l}=0 \text { and } M^{l}=0
$$

Where $l=1$ at $\mathrm{x}=0$, while $l=\mathrm{n}+1$ at $\mathrm{x}=\mathrm{L}$.

At the cracked locations, the boundary conditions can be

should be considered for EBT, while

$$
\Phi^{l+1}-\Phi^{l}=K^{l} \frac{d \Phi^{l}}{d x}
$$

should be considered with TBT. Where $K^{l}$ is crack-severity of $l$-th crack, $(l=1, \mathrm{n})$,

$M^{l}$ denotes to the bending moment of sub-beam $l, Q^{l}$ denotes to the shear force of sub-beam $l,(l=1, \mathrm{n}+1)$,

$$
\begin{aligned}
& M^{l}=-\frac{E^{l} I^{l}}{\left(L^{l}\right)^{2}}\left[\frac{d^{2} w^{l}}{d x^{2}}+\omega^{2}\left(e_{0}^{l} a^{l}\right)^{2} \rho^{l} A^{l} \frac{\left(L^{l}\right)^{2}}{E^{l} I^{l}} w^{l}\right] \\
& Q^{l}=-\frac{E^{l} I^{l}}{\left(L^{l}\right)^{3}}\left[\frac{d^{3} w^{l}}{d x^{3}}+\omega^{2}\left(e_{0}^{l} a^{l}\right)^{2} \rho^{l} A^{l} \frac{\left(L^{l}\right)^{2}}{E^{l} I^{l}} \frac{d w^{l}}{d x}\right]
\end{aligned}
$$

For EBT as [27], While 


$$
\begin{gathered}
M^{l}=E^{l} I^{l} \frac{d \phi^{l}}{d x}-\omega^{2}\left(e_{0}^{l} a^{l}\right)^{2}\left(\rho^{l} A^{l} w^{l}+\rho^{l} I^{l} \frac{d \phi^{l}}{d x}\right) \\
Q^{l}=K_{s}^{l} G^{l} A^{l}\left(\Phi^{l}+\frac{d w^{l}}{d x}\right)
\end{gathered}
$$

For TBT as [8], Where $L^{l}$ denotes to the length of subbeaml, $(l=1, \mathrm{n}+1)$.

$\Phi^{l}$ is the rotational angle in TBT, while it represents to $\frac{d w^{l}}{d x}$ in EBT.
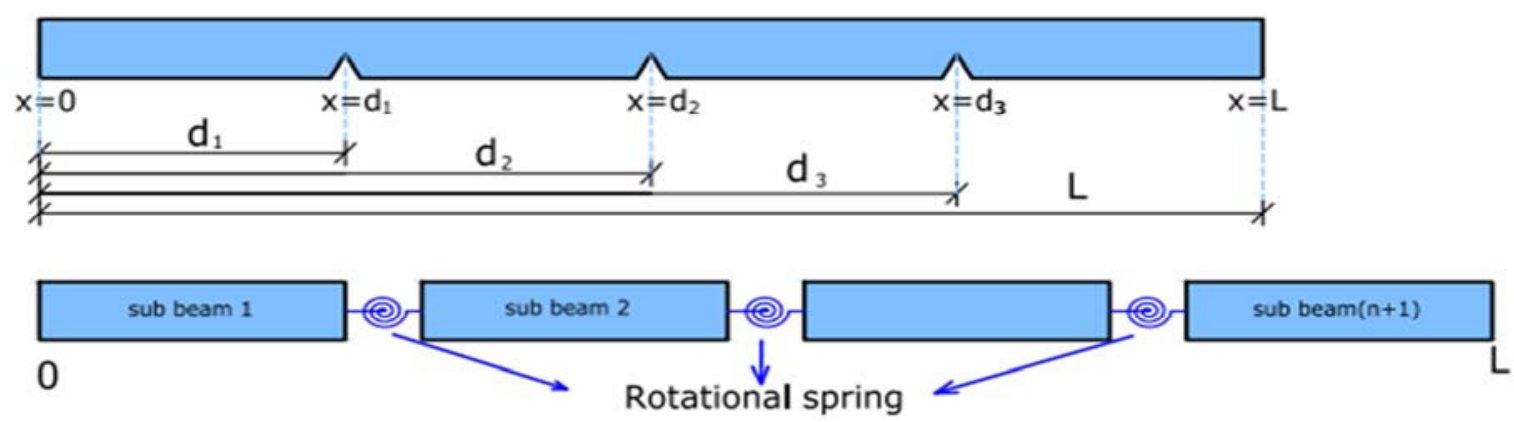

Figure 1. Model of cracked beam.

\section{Method of Solution}

Polynomial based differential quadrature method (PDQM) is employed to reduce the problem to that of linear algebraic system. The derivatives of field quantities are approximated as [28-31]:

$$
\left.\frac{\partial^{m} f(x)}{\partial x^{m}}\right|_{x=x_{i}}=\sum_{j=1}^{N} C_{i j}^{m} f\left(x_{j}\right),(\mathrm{i}=1, \mathrm{~N}),(\mathrm{m}=1, \mathrm{M}) .
$$

Where $\mathrm{N}$ is the number of the nodes for each sub-beam, $\mathrm{M}$ is the order of the highest derivative appearing in the problem, $\mathrm{f}\left(\mathrm{x}_{i}\right)$ is the nodal values of field quantities at the points $x_{i},(\mathrm{i}=1, \mathrm{~N}) ; \mathrm{N}>\mathrm{M}$.

$\mathrm{C}_{\mathrm{ij}}^{\mathrm{m}},(\mathrm{i}, \mathrm{j}=1, \mathrm{~N})$, are the weighting coefficients relating the mth derivative to the functional values at $x_{i}$.

For first order derivative, the weighting coefficients can be expressed as [28-31]:

$$
\begin{aligned}
C_{i j}^{1}= & \left\{\begin{array}{cc}
\frac{1}{x_{i}-x_{j}}\left(\frac{P_{i}}{P_{j}}\right), i \neq j & \begin{array}{l}
\text { one can reduce the problem to the follow } \\
\text { system: } \\
-\sum_{j=1, j \neq i}^{N} C_{i j}^{1}, i=j
\end{array} \\
& \sum_{j=1}^{N} C_{i j}^{4} w_{j}^{l}=\omega^{2} \frac{\rho^{l} A^{l}}{E^{l} I^{l}}\left[w_{i}^{l}-\left(e_{0}^{l} a^{l}\right)^{2} \sum_{j=1}^{N} C_{i j}^{2} w_{j}^{l}\right](\mathrm{i}, \mathrm{j}=1,2, \ldots, \mathrm{N}),(l=1, \mathrm{n}+1) .
\end{array}\right.
\end{aligned}
$$

Where $P_{i}$ is defined as:

$$
P_{i}=\prod_{j=1, j \neq i}^{N}\left(x_{i}-x_{j}\right), i, j=1,2, \ldots, N
$$

For second order derivative, the weighting coefficients can be expressed as:

$$
C_{i j}^{2}=\left\{\begin{array}{c}
2 C_{i j}^{1}\left(C_{i i}^{1}-\frac{1}{x_{i}-x_{j}}\right), i, j=1,2, \ldots, N, i \neq j \\
-\sum_{j=1, j \neq i}^{N} C_{i j}^{2}, i=1,2, \ldots, N, i=j,
\end{array}\right.
$$

The distribution of the nodes $x_{i}$ is calculated according to Chebyshev-Gauss-Lobatto discretization as [28-31]:

$$
x_{i}=x_{1}+\frac{x_{N}-x_{1}}{2}\left(1-\cos \left(\frac{i-1}{N-1} \pi\right)\right), i=1,2, \ldots, N
$$

\subsection{Solution of Euler-Bernoulli Problem}

Applying prescribed PDQM to equations (2) and (5-11), one can reduce the problem to the following linear algebraic

The boundary conditions at external boundaries, $(\mathrm{x}=0$ and $\mathrm{x}=\mathrm{L})$ can be written as follows:

For a simply supported end:

$$
w_{i}^{l}=0, \sum_{j=1}^{N} C_{i j}^{2 l} w_{j}^{l}+\omega^{2}\left(e_{0}^{l} a^{l}\right)^{2} \frac{\rho^{l} A^{l} L^{l^{2}}}{E^{l} I^{l}} w_{i}^{l}=0
$$

For a clamped supported end:

$$
w_{i}^{l}=0, \sum_{j=1}^{N} C_{i j}^{1}{ }^{l} w_{j}^{l}=0
$$

For a free supported end:

$$
\sum_{j=1}^{N} C_{i j}^{3^{l}} w_{j}^{l}+\omega^{2}\left(e_{0}^{l} a^{l}\right)^{2} \frac{\rho^{l} A^{l} L^{l^{2}}}{E^{l} I^{l}} \sum_{j=1}^{N} C_{i j}^{1}{ }^{l} w_{j}^{l}=0, \sum_{j=1}^{N} C_{i j}^{2 l} w_{j}^{l}+\omega^{2}\left(e_{0}^{l} a^{l}\right)^{2} \frac{\rho^{l} A^{l} L^{L^{2}}}{E^{l} I^{l}} w_{i}^{l}=0
$$

Where $l=1$ at $x=0$ and $l=n+1$ at $x=L$.

At crack sites $\left(\mathrm{x}=d_{l},(l=1, \mathrm{n})\right)$, the following conditions should be considered: 


$$
\begin{gathered}
\mathrm{w}_{N}^{l}=\mathrm{w}_{1}^{l+1} \\
\frac{E^{l} I^{l}}{L^{l}}\left[\sum_{j=1}^{N} C_{N j}^{2} w_{j}^{l}+\omega^{2}\left(e_{0}^{l} a^{l}\right)^{2} \frac{\rho^{l} A^{l}\left(L^{l}\right)^{2}}{E^{l} I^{l}} w_{N}^{l}\right]=\frac{E^{l+1} I^{l+1}}{L^{l+1}}\left[\sum_{j=1}^{N} C_{1 j}^{2} w_{j}^{l+1}+\omega^{2}\left(e_{0}^{l+1} a^{l+1}\right)^{2} \frac{\rho^{l+1} A^{l+1}\left(L^{l+1}\right)^{2}}{E^{l+1} I^{l+1}} w_{1}^{l+1}\right] \\
\frac{E^{l} I^{l}}{\left(L^{l}\right)^{3}}\left[\sum_{j=1}^{N} C_{N j}^{3} w_{j}^{l}+\omega^{2}\left(e_{0}^{l} a^{l}\right)^{2} \frac{\rho^{l} A^{l} L^{l^{2}}}{E^{l} I^{l}} \sum_{j=1}^{N} C_{N j}^{1} w_{j}^{l}\right] \\
=\frac{E^{l+1} I^{l+1}}{\left(L^{l+1}\right)^{3}}\left[\sum_{j=1}^{N} C_{1 j}^{3} w_{j}^{l+1}+\omega^{2}\left(e_{0}^{l+1} a^{l+1}\right)^{2} \frac{\rho^{l+1} A^{l+1}\left(L^{l+1}\right)^{2}}{E^{l+1} I^{l+1}} \sum_{j=1}^{N} C_{1 j}^{1} w_{j}^{l+1}\right] \\
\sum_{j=1}^{N} C_{1 j}^{1} w_{j}^{l+1}-\sum_{j=1}^{N} C_{N j}^{1} w_{j}^{l}=K^{l} \sum_{j=1}^{N} C_{N j}^{2} w_{j}^{l}
\end{gathered}
$$

\subsection{Solution of Timoshenko Problem}

Also, by applying PDQM to equations (3, 4), (5-10) and (12), one can reduce the problem to the following linear algebraic system:

$$
\begin{gathered}
{\left[E^{l} I^{l}-\omega^{2}\left(e_{0}^{l} a^{l}\right)^{2} \rho^{l} I^{l}\right] \sum_{j=1}^{N} C_{i j}^{2} \phi_{j}^{l}+\left[\omega^{2} \rho^{l} I^{l}-K_{s}^{l} G^{l} A^{l}\right] \phi_{i}^{l}-\left[\omega^{2}\left(e_{0}^{l} a^{l}\right)^{2} \rho^{l} A^{l}+K_{s}^{l} G^{l} A^{l}\right] \sum_{j=1}^{N} C_{i j}^{1} w_{j}^{l}=0} \\
K_{S}^{l} G^{l} A^{l}\left(\sum_{j=1}^{N} C_{i j}^{1} \phi_{j}^{l}+\sum_{j=1}^{N} C_{i j}^{2} w_{j}^{l}\right)+\omega^{2} \rho^{l} A^{l} w_{i}^{l}=0
\end{gathered}
$$

The boundary conditions at external boundaries, $(\mathrm{x}=0$ and $\mathrm{x}=\mathrm{L})$ can be written as follows:

For a simply supported end:

$$
w_{i}^{l}=0,\left[E^{l} I^{l}-\omega^{2}\left(e_{0}^{l} a^{l}\right)^{2} \rho^{l} I^{l}\right] \sum_{j=1}^{N} C_{i j}^{1} \phi_{j}^{l}-\omega^{2}\left(e_{0}^{l} a^{l}\right)^{2} \rho^{l} A^{l} w_{i}^{l}=0
$$

For a clamped supported end:

$$
w_{i}^{l}=0, \Phi_{i}^{l}=0
$$

For a free supported end:

$$
K_{S}^{l} G^{l} A^{l}\left(\phi_{i}^{l}+\sum_{j=1}^{N} C_{i j}^{1} w_{j}^{l}\right)=0,\left[E^{l} I^{l}-\omega^{2}\left(e_{0}^{l} a^{l}\right)^{2} \rho^{l} I^{l}\right] \sum_{j=1}^{N} C_{i j}^{1} \phi_{j}^{l}-\omega^{2}\left(e_{0}^{l} a^{l}\right)^{2} \rho^{l} A^{l} w_{i}^{l}=0
$$

Where $l=1$ at $\mathrm{x}=0$, while $l=\mathrm{n}+1$ at $\mathrm{x}=\mathrm{L}$.

At crack sites $\left(\mathrm{x}=\mathrm{d}_{\mathrm{l}},(l=1, \mathrm{n})\right)$, the following conditions should be considered:

$$
\begin{gathered}
w_{N}^{l}=w_{1}^{l+1} \\
E^{l} I^{l} \sum_{j=1}^{N} C_{N j}^{1} \phi_{j}^{l}-\omega^{2}\left(e_{0}^{l} a^{l}\right)^{2}\left(\rho^{l} A^{l} w_{N}^{l}+\rho^{l} I^{l} \sum_{j=1}^{N} C_{N j}^{1} \phi_{j}^{l}\right) \\
=E^{l+1} I^{l+1} \sum_{j=1}^{N} C_{1 j}^{1} \phi_{j}^{l+1}-\omega^{2}\left(e_{0}^{l+1} a^{l+1}\right)^{2}\left(\rho^{l+1} A^{l+1} w_{1}^{l+1}+\rho^{l+1} I^{l+1} \sum_{j=1}^{N} C_{1 j}^{1} \phi_{j}^{l+1}\right) \\
K_{s}^{l} G^{l} A^{l}\left(\Phi_{N}^{l}+\sum_{j=1}^{N} C_{N j}^{1} w_{j}^{l}\right)=K_{s}^{l+1} G^{l+1} A^{l+1}\left(\Phi_{1}^{l+1}+\sum_{j=1}^{N} C_{1 j}^{1} w_{j}^{l+1}\right) \\
\Phi_{1}^{l+1}-\Phi_{N}^{l}=K^{l} \sum_{j=1}^{N} C_{N j}^{1} \phi_{j}^{l}
\end{gathered}
$$

Consider a numerical example of single wall carbon nanotube with diameter $\mathrm{d}=0.678 \mathrm{~nm}$, length $\mathrm{L}=10 \mathrm{~d}$, Poisson's ratio $\mathrm{v}=0.19$, and have effective tube thickness $\mathrm{t}=$ $0.066 \mathrm{~nm}$ and $\mathrm{E}=5.5 \mathrm{TPa}$ as given in [8].

\section{Numerical Results}

For practical purpose, all field quantities are normalized such as:

$$
\bar{x}=\frac{x}{L}, \bar{w}^{l}=\frac{w^{l}}{L}, \lambda^{2}=\omega^{2} \frac{\rho A L^{4}}{E I}, \Omega=\frac{E I}{K_{S} G A L^{2}}, \alpha=\frac{\left(e_{0} a\right)}{L}, \xi=L \sqrt{\frac{A}{I}}
$$

Where $L$ is the length of the whole beam, $\lambda^{2}$ is the frequency parameter, $\Omega$ is the shear deformation parameter, $\alpha$ is the scaling effect parameter and $\xi$ is the slenderness ratio.

To ensure the validity of proposed scheme, the obtained results are compared with previous ones for cracked and un- 
cracked Euler- Bernoulli and Timoshenko problems. A quadrature numerical scheme is designed to solve cracked Euler- Bernoulli beam problems, equations (22-29). For each sub-beam, $\mathrm{N}$ is to be varied from 5-50 to determine $\mathrm{N}$ leading to accurate convergent results. As well as another scheme is designed for cracked Timoshenko beams. Tables (1-3) show the convergence of the obtained results compared with the previous ones for different supporting conditions. Further a parametric study is introduced using $\mathrm{N}=10$ to investigate influence of crack severity $\mathrm{K}$, scaling effect parameter $\alpha$ and crack location $\bar{x}$ on the obtained results.

Table 1 compares the obtained results for simply supported Euler- Bernoulli nanobeam with Loya [16], while it compares the obtained results for Timoshenko nanobeam with Torabi [21]. The obtained results for Timoshenko nanobeam are very accurate with the exact solution more than Torabi [21] as shown in Table 1. Tables 2, 3 show the obtained results for clamped-clamped, clamped simply supported and clamped-free nanobeam. Figures 2, 3, 4, 5, 6, 7 show the influence of crack severity K, scaling effect parameter $\alpha$ and crack position $\bar{x}$ on fundamental frequency parameter $\sqrt{\lambda}$.
The figures show that:

The value of fundamental frequency parameter $\sqrt{\lambda}$, decreases with the increasing of crack severity $\mathrm{K}$, as shown in Figures $(2,3)$. Also, the value of fundamental frequency parameter $\sqrt{\lambda}$ decreases with the increasing of scaling effect parameter $\alpha$ as shown in Figures $(4,5)$. The location of the crack effects on the value of fundamental frequency parameter $\sqrt{\lambda}$ as follows:

In simply supported beam, the values of fundamental frequency parameter $\sqrt{\lambda}$ are reduced as much as crack location moves away from the support and reach the minimum when the crack is in the middle of the span. While in clamped-clamped beam, the values of fundamental frequency parameter $\sqrt{\lambda}$ are increased as much as crack location moves away from the support and reach the maximum when the place of the crack almost reaches $\overline{\mathrm{x}}=0.25, \overline{\mathrm{x}}=0.75$ then decreasing to reach the minimum when the crack almost is in the middle of the span. While in clamped-simple beam, the values of fundamental frequency parameter $\sqrt{\lambda}$ are decreased as much as crack location moves away from the simple support, but it are increased as much as crack location moves away from the clamped support as shown in Figures $(6,7)$.

Table 1. Comparison of the obtained frequencies $\sqrt{\lambda}$, for simply supported nano-beam, with the previous results: crack-severity $K=0$, crack site at $\bar{x}=0.5$.

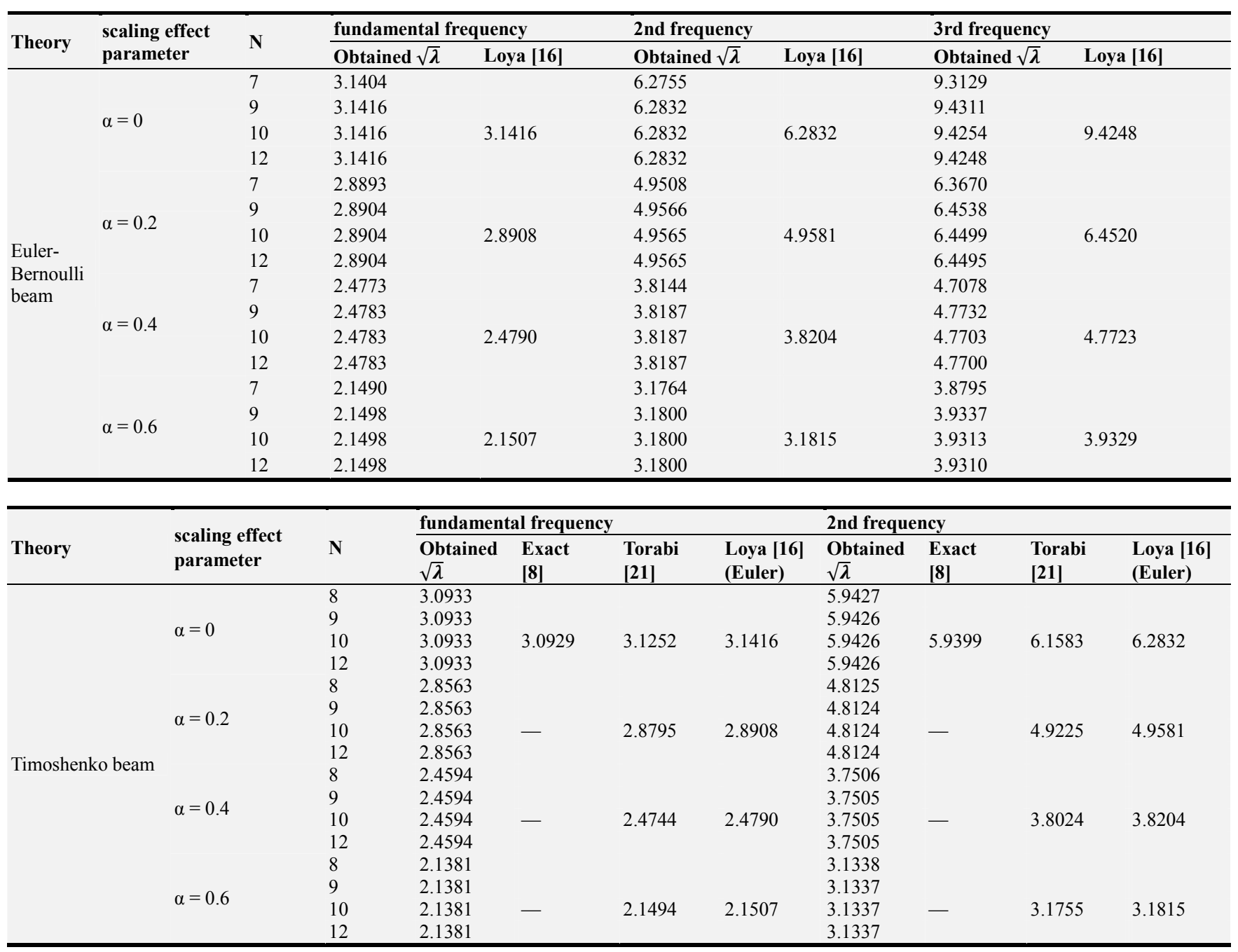




\begin{tabular}{|c|c|c|c|c|c|c|}
\hline \multirow[b]{2}{*}{ Theory } & \multirow{2}{*}{$\begin{array}{l}\text { scaling effect } \\
\text { parameter }\end{array}$} & \multirow[b]{2}{*}{$\mathbf{N}$} & \multicolumn{4}{|l|}{ 3rd frequency } \\
\hline & & & Obtained $\sqrt{\lambda}$ & $\begin{array}{l}\text { Exact } \\
{[8]} \\
\end{array}$ & $\begin{array}{l}\text { Torabi } \\
\text { [21] }\end{array}$ & $\begin{array}{l}\begin{array}{l}\text { Loya [16] } \\
\text { (Euler) }\end{array} \\
\end{array}$ \\
\hline \multirow{16}{*}{ Timoshenko beam } & \multirow{4}{*}{$\alpha=0$} & 8 & 8.4536 & \multirow{5}{*}{8.4444} & \multirow{5}{*}{9.0328} & \multirow{4}{*}{9.4248} \\
\hline & & 9 & 8.4509 & & & \\
\hline & & 10 & 8.4512 & & & \\
\hline & & 12 & 8.4512 & & & \\
\hline & \multirow{4}{*}{$\alpha=0.2$} & 8 & 6.1605 & & & \multirow{4}{*}{6.4520} \\
\hline & & 9 & 6.1584 & \multirow{3}{*}{ - } & \multirow{3}{*}{6.4222} & \\
\hline & & 10 & 6.1586 & & & \\
\hline & & 12 & 6.1586 & & & \\
\hline & \multirow{4}{*}{$\alpha=0.4$} & 8 & 4.6210 & \multirow{4}{*}{ - } & \multirow{5}{*}{4.6087} & \multirow{4}{*}{4.7723} \\
\hline & & 9 & 4.6194 & & & \\
\hline & & 10 & 4.6196 & & & \\
\hline & & 12 & 4.6196 & & & \\
\hline & \multirow{4}{*}{$\alpha=0.6$} & 8 & 3.8210 & \multirow{4}{*}{ - } & & \multirow{4}{*}{3.9329} \\
\hline & & 9 & 3.8197 & & \multirow{3}{*}{3.9212} & \\
\hline & & 10 & 3.8198 & & & \\
\hline & & 12 & 3.8198 & & & \\
\hline
\end{tabular}

Table 2. Comparison of the obtained frequencies $\sqrt{\lambda}$, for clamped-clamped nano- beam, with the previous results: crack-severity $K=0.35$, crack site at $\bar{x}=0.5$.

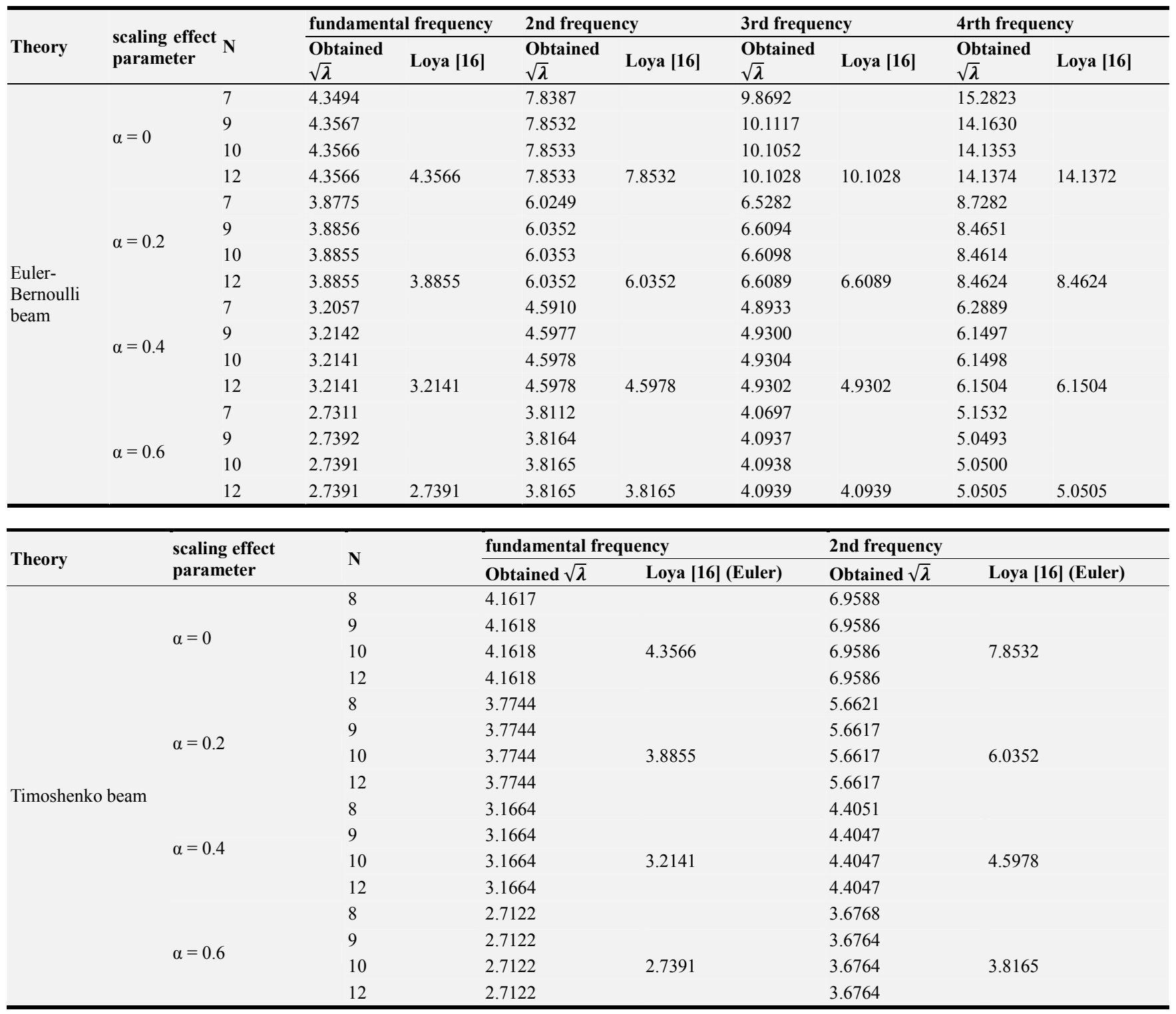




\begin{tabular}{|c|c|c|c|c|c|c|}
\hline \multirow{2}{*}{ Theory } & \multirow{2}{*}{$\begin{array}{l}\text { scaling effect } \\
\text { parameter }\end{array}$} & \multirow{2}{*}{$\mathbf{N}$} & \multicolumn{2}{|l|}{ 3rd frequency } & \multicolumn{2}{|c|}{ 4rth frequency } \\
\hline & & & Obtained $\sqrt{\lambda}$ & Loya [16] (Euler) & Obtained $\sqrt{\lambda}$ & Loya [16] (Euler) \\
\hline \multirow{16}{*}{ Timoshenko beam } & \multirow{4}{*}{$\alpha=0$} & 8 & 8.6241 & \multirow{5}{*}{10.1028} & 11.1318 & \multirow{5}{*}{14.1372} \\
\hline & & 9 & 8.6207 & & 11.1290 & \\
\hline & & 10 & 8.6210 & & 11.1280 & \\
\hline & & 12 & 8.6210 & & 11.1282 & \\
\hline & \multirow{4}{*}{$\alpha=0.2$} & 8 & 6.2240 & & 7.6826 & \\
\hline & & 9 & 6.2221 & \multirow{3}{*}{6.6089} & 7.6799 & \multirow{4}{*}{8.4624} \\
\hline & & 10 & 6.2221 & & 7.6783 & \\
\hline & & 12 & 6.2222 & & 7.6785 & \\
\hline & \multirow{4}{*}{$\alpha=0.4$} & 8 & 4.7373 & \multirow{4}{*}{4.9302} & 5.6879 & \\
\hline & & 9 & 4.7365 & & 5.6860 & \multirow{4}{*}{6.1504} \\
\hline & & 10 & 4.7365 & & 5.6846 & \\
\hline & & 12 & 4.7365 & & 5.6847 & \\
\hline & \multirow{4}{*}{$\alpha=0.6$} & 8 & 3.9576 & \multirow{4}{*}{4.0939} & 4.6893 & \\
\hline & & 9 & 3.9572 & & 4.6878 & \multirow{3}{*}{5.0505} \\
\hline & & 10 & 3.9572 & & 4.6866 & \\
\hline & & 12 & 3.9572 & & 4.6867 & \\
\hline
\end{tabular}

Table 3. Comparison of the obtained frequencies $\sqrt{\lambda}$, for Clamped-simply supported and Clamped-free Euler-Bernoulli nano-beam, with the previous results: crack-severity $K=0.35$, crack site at $\bar{x}=0.5$.

\begin{tabular}{|c|c|c|c|c|c|c|}
\hline \multirow{2}{*}{ boundary condition } & \multirow{2}{*}{$\begin{array}{l}\text { scaling effect } \\
\text { parameter }\end{array}$} & \multirow{2}{*}{$\mathbf{N}$} & \multicolumn{2}{|c|}{ fundamental frequency } & \multicolumn{2}{|l|}{ 2nd frequency } \\
\hline & & & Obtained $\sqrt{\lambda}$ & Bahrami [19] & Obtained $\sqrt{\lambda}$ & Bahrami [19] \\
\hline \multirow{16}{*}{$\begin{array}{l}\text { clamped-simply } \\
\text { supported }\end{array}$} & \multirow{4}{*}{$\alpha=0$} & 7 & 3.6155 & \multirow{4}{*}{3.6195} & 6.9480 & \multirow{4}{*}{6.9700} \\
\hline & & 9 & 3.6182 & & 6.9660 & \\
\hline & & 10 & 3.6181 & & 6.9657 & \\
\hline & & 12 & 3.6181 & & 6.9657 & \\
\hline & \multirow{4}{*}{$\alpha=0.2$} & 7 & 3.2742 & \multirow{4}{*}{3.2780} & 5.3204 & \multirow{4}{*}{5.3428} \\
\hline & & 9 & 3.2768 & & 5.3393 & \\
\hline & & 10 & 3.2768 & & 5.3390 & \\
\hline & & 12 & 3.2768 & & 5.3390 & \\
\hline & \multirow{4}{*}{$\alpha=0.4$} & 7 & 2.7563 & \multirow{5}{*}{2.7597} & 4.0345 & \multirow{5}{*}{4.0523} \\
\hline & & 9 & 2.7587 & & 4.0495 & \\
\hline & & 10 & 2.7587 & & 4.0493 & \\
\hline & & 12 & 2.7587 & & 4.0492 & \\
\hline & \multirow{4}{*}{$\alpha=0.6$} & 7 & 2.3689 & & 3.3439 & \\
\hline & & 9 & 2.3711 & \multirow{3}{*}{2.3719} & 3.3561 & \multirow{3}{*}{3.3585} \\
\hline & & 10 & 2.3711 & & 3.3560 & \\
\hline & & 12 & 2.3711 & & 3.3559 & \\
\hline \multirow{12}{*}{ Clamped-free } & \multirow{4}{*}{$\alpha=0$} & 7 & 1.8032 & \multirow{4}{*}{1.8052} & 4.1505 & \\
\hline & & 9 & 1.8033 & & 4.1575 & 4.1607 \\
\hline & & 10 & 1.8033 & & 4.1574 & \\
\hline & & 12 & 1.8033 & & 4.1574 & \\
\hline & & 7 & 1.8241 & & 3.6243 & \\
\hline & & 9 & 1.8243 & 1.8263 & 3.6333 & 3.6352 \\
\hline & $\alpha=0.2$ & 10 & 1.8243 & & 3.6332 & \\
\hline & & 12 & 1.8243 & & 3.6332 & \\
\hline & & 7 & 1.9104 & & 2.8223 & \\
\hline & & 9 & 1.9109 & 1.9135 & 2.8276 & 2.8280 \\
\hline & $\alpha=0.4$ & 10 & 1.9109 & & 2.8276 & \\
\hline & & 12 & 1.9109 & & 2.8276 & \\
\hline & & & Table 3. Cor & & & \\
\hline boundary condition & scaling effect & $\mathbf{N}$ & 3rd frequency & & 4rth frequency & \\
\hline 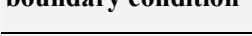 & parameter & & Obtained $\sqrt{\lambda}$ & Bahrami [19] & Obtained $\sqrt{\lambda}$ & Bahrami [19] \\
\hline & & 7 & 9.3292 & & 14.1368 & \\
\hline & & 9 & 9.4805 & & 13.2023 & \\
\hline & $\alpha=0$ & 10 & 9.4756 & 9.4775 & 13.1930 & 13.1997 \\
\hline & & 12 & 9.4744 & & 13.1908 & \\
\hline & & 7 & 6.3419 & & 8.1943 & \\
\hline & & 9 & 6.3943 & 6.3945 & 7.7840 & 7.7923 \\
\hline & $\alpha=0.2$ & 10 & 6.3941 & & 7.7864 & \\
\hline clamped-simply & & 12 & 6.3937 & & 7.7851 & \\
\hline supported & & 7 & 4.7653 & & 5.9213 & \\
\hline & & 9 & 4.7869 & & 5.6381 & \\
\hline & $\alpha=0.4$ & 10 & 4.7871 & 4.7874 & 5.6388 & 5.6436 \\
\hline & & 12 & 4.7870 & & 5.6383 & \\
\hline & & 7 & 3.9574 & & 4.8549 & \\
\hline & & 9 & 3.9706 & & 4.6266 & \\
\hline & $\alpha=0.6$ & 10 & 3.9707 & 3.9710 & 4.6268 & 4.6307 \\
\hline & & 12 & 3.9707 & & 4.6264 & \\
\hline
\end{tabular}




\begin{tabular}{|c|c|c|c|c|c|c|}
\hline \multirow{2}{*}{ boundary condition } & \multirow{2}{*}{$\begin{array}{l}\text { scaling effect } \\
\text { parameter }\end{array}$} & \multirow{2}{*}{$\mathbf{N}$} & \multicolumn{2}{|l|}{ 3rd frequency } & \multicolumn{2}{|l|}{ 4rth frequency } \\
\hline & & & Obtained $\sqrt{\lambda}$ & Bahrami [19] & Obtained $\sqrt{\lambda}$ & Bahrami [19] \\
\hline \multirow{12}{*}{ Clamped-free } & \multirow{4}{*}{$\alpha=0$} & 7 & 7.8016 & \multirow{4}{*}{7.8541} & 9.8328 & \multirow{4}{*}{10.1175} \\
\hline & & 9 & 7.8480 & & 10.1186 & \\
\hline & & 10 & 7.8468 & & 10.1118 & \\
\hline & & 12 & 7.8465 & & 10.1077 & \\
\hline & \multirow{4}{*}{$\alpha=0.2$} & 7 & 5.9285 & \multirow{5}{*}{6.0618} & 6.4417 & \multirow{5}{*}{6.6733} \\
\hline & & 9 & 6.0629 & & 6.6839 & \\
\hline & & 10 & 6.0589 & & 6.6732 & \\
\hline & & 12 & 6.0583 & & 6.6687 & \\
\hline & \multirow{4}{*}{$\alpha=0.4$} & 7 & 4.4421 & & 4.7375 & \\
\hline & & 9 & - & \multirow[t]{3}{*}{ - } & - & \multirow[t]{3}{*}{ - } \\
\hline & & 10 & - & & - & \\
\hline & & 12 & - & & - & \\
\hline
\end{tabular}

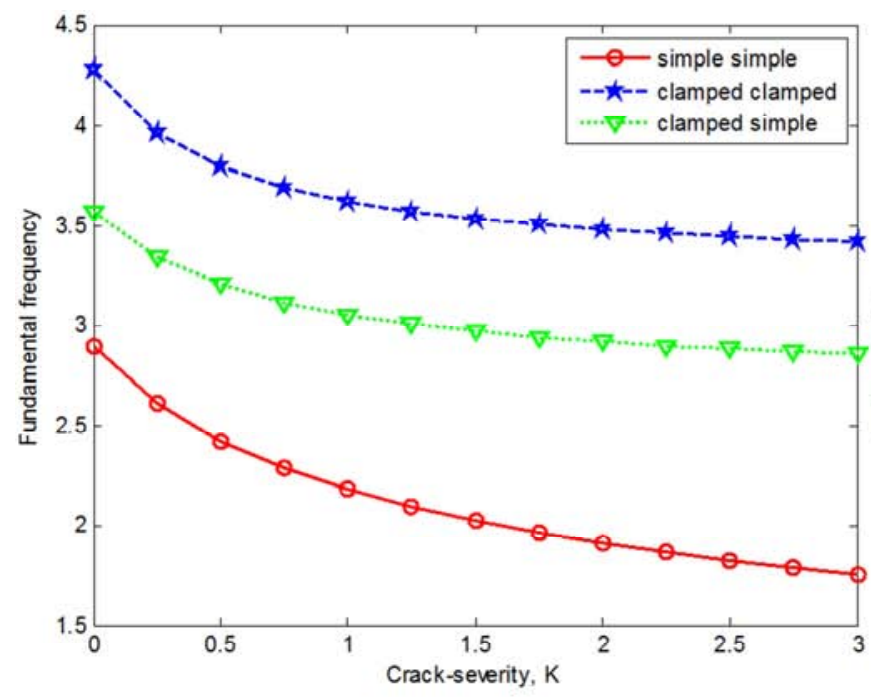

$\alpha=0.2$

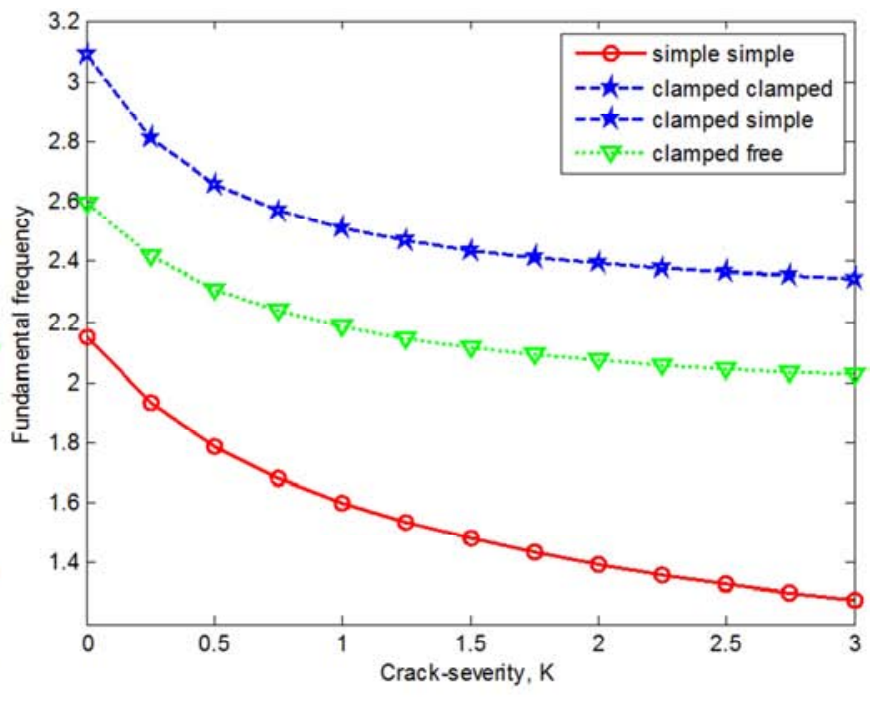

$\alpha=0.6$

Figure 2. Variation of the obtained fundamental frequency $\sqrt{\lambda}$ with the crack severity $K$, for Euler-Bernoulli beam subjected to different supporting conditions and different scaling effect parameter $\alpha$ : crack site at $\bar{x}=0.5$.

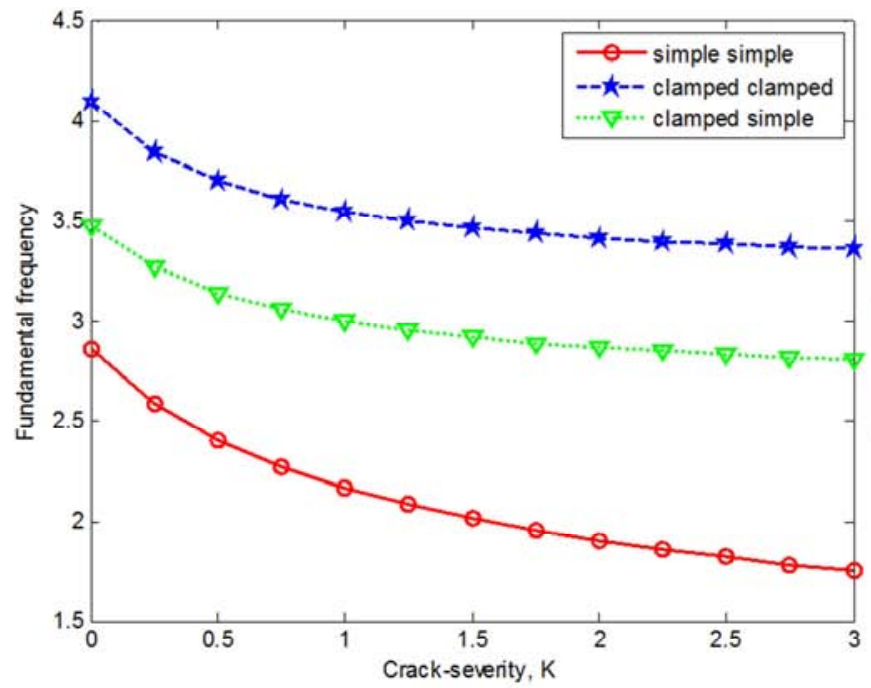

$\alpha=0.2$

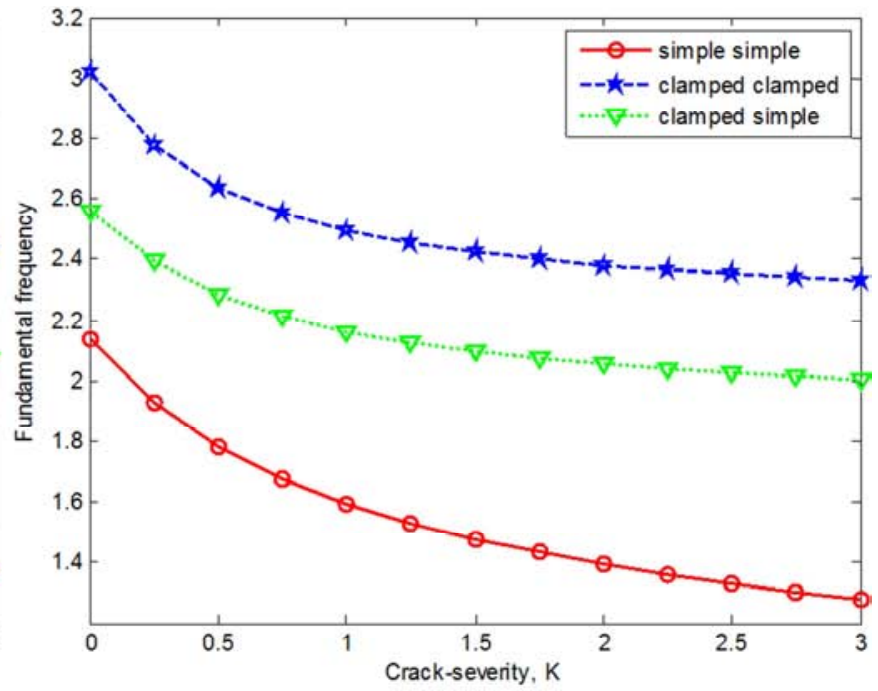

$\alpha=0.6$

Figure 3. Variation of the obtained fundamental frequency $\sqrt{\lambda}$ with the crack severity $K$, for Timoshenko beam subjected to different supporting conditions and different scaling effect parameter $\alpha$ : crack site at $\bar{x}=0.5$. 


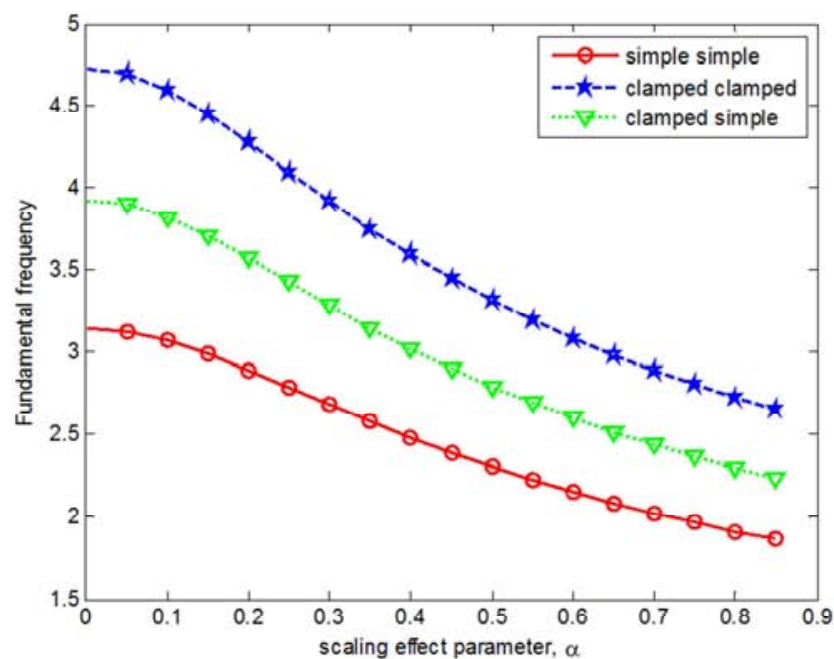

$\mathrm{k}=0$

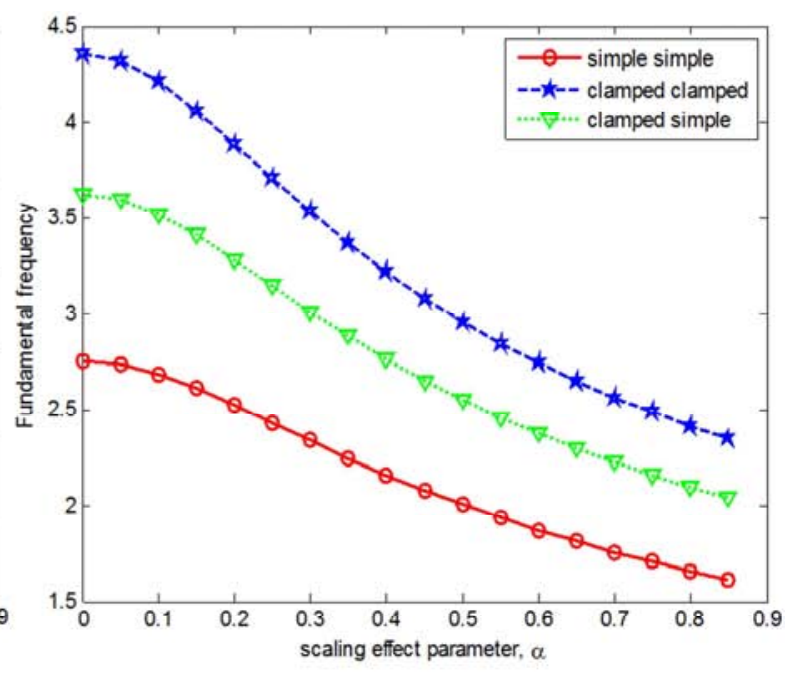

$\mathrm{k}=0.35$

Figure 4. Variation of the obtained fundamental frequency $\sqrt{\lambda}$ with scaling effect parameter $\alpha$, for Euler-Bernoulli beam subjected to different supporting conditions and different crack severity $K$ : crack site at $\bar{x}=0.5$.

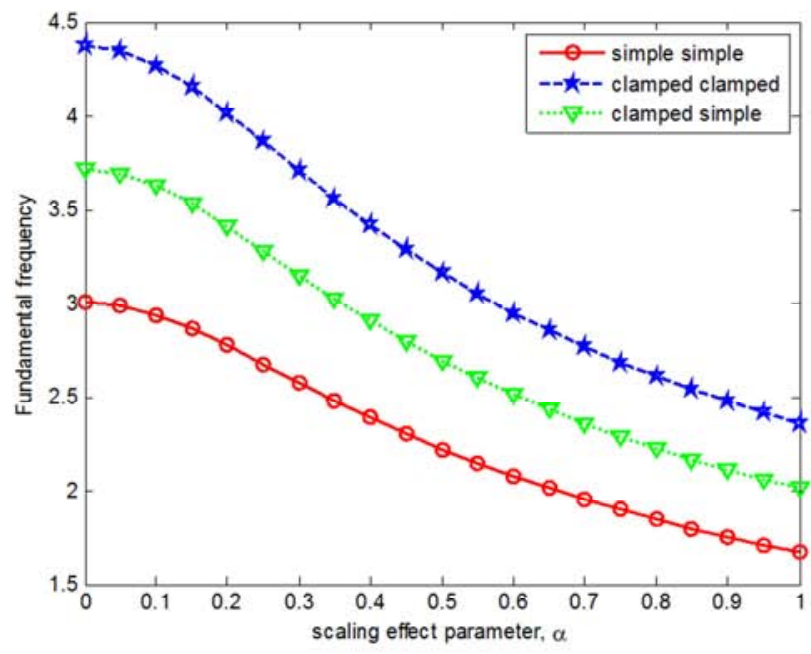

$\mathrm{k}=0.065$

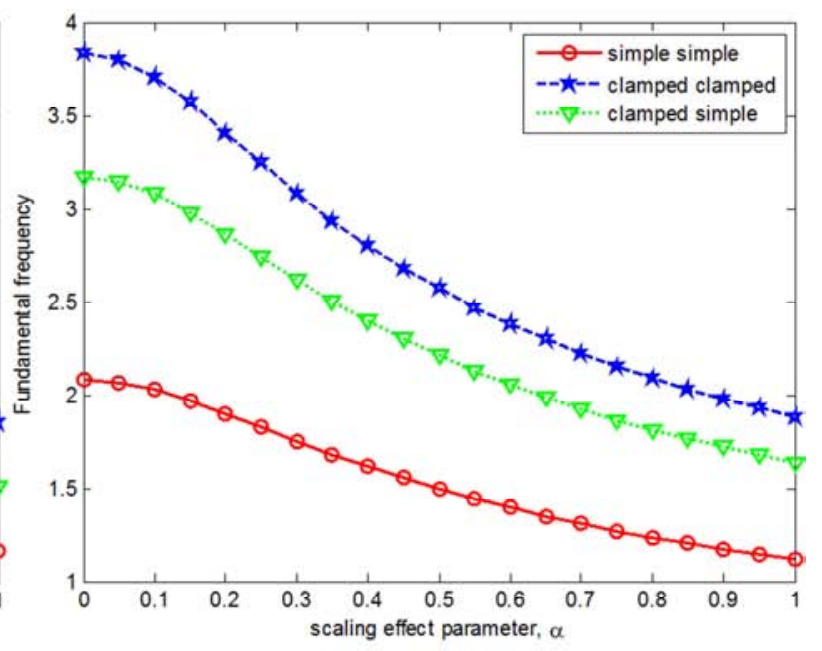

$\mathrm{k}=2$

Figure 5. Variation of the obtained fundamental frequency $\sqrt{\lambda}$ with scaling effect parameter $\alpha$, for Timoshenko beam subjected to different supporting conditions and different crack severity $K$ : crack site at $\bar{x}=0.5$.

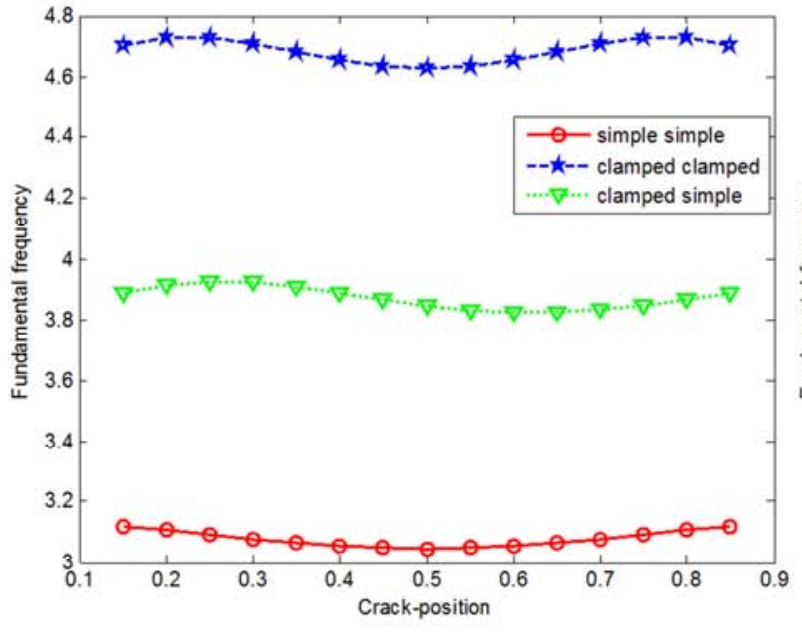

$\mathrm{k}=0.065$

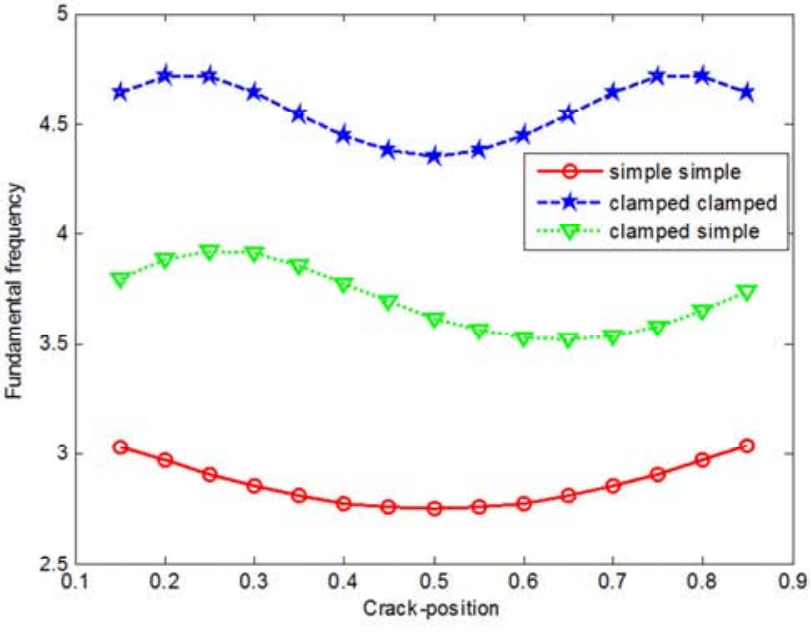

$\mathrm{k}=0.35$

Figure 6. Variation of the obtained fundamental frequency $\sqrt{\lambda}$ with the crack position $\bar{x}$, for Euler-Bernoulli beam subjected to different supporting conditions and different crack severity $K$ : scaling effect parameter $\alpha=0$. 

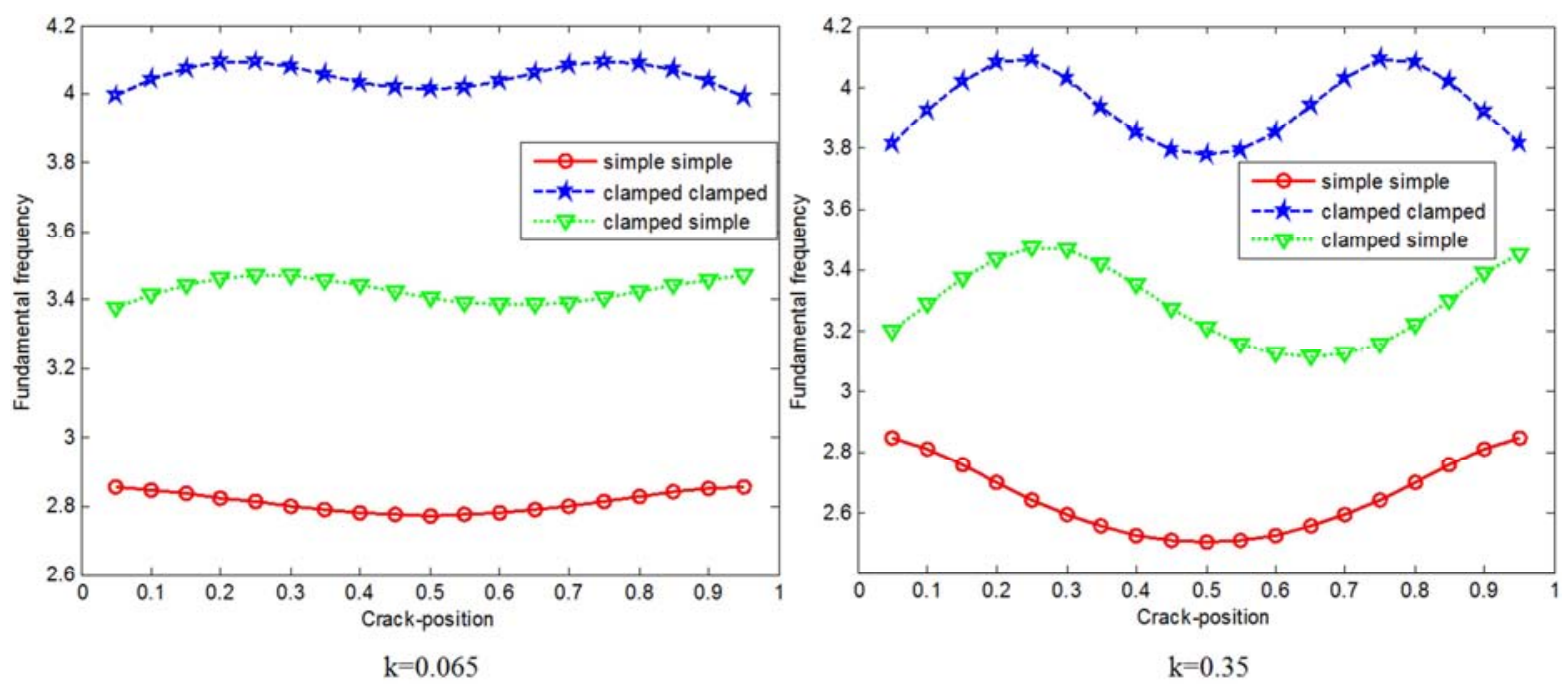

Figure 7. Variation of the obtained fundamental frequency $\sqrt{\lambda}$ with the crack position $\bar{x}$, for Timoshenko beam subjected to different supporting conditions and different crack severity $K$ : scaling effect parameter $\alpha=0.2$.

\section{Conclusion}

A numerical scheme based on differential quadrature method, is successfully examined for free vibration analysis of cracked Euler-Bernoulli beam and Timoshenko beam. The obtained results agreed with previous ones of $\mathrm{N}=10$. Further a parametric study is employed to investigate the influence of crack severity $\mathrm{K}$, scaling effect parameter $\alpha$ and crack location on the obtained results.

a. The value of fundamental frequency parameter $\sqrt{\lambda}$ decreases with the increasing of crack severity $\mathrm{K}$.

b. The value of fundamental frequency parameter $\sqrt{\lambda}$ decreases with the increasing of scaling effect parameter $\alpha$.

c. The influence of crack severity $\mathrm{K}$ is very small on frequency parameter $\sqrt{\lambda}$, when the crack locates near to the clamped support.

It is aimed that these results may be useful for design purposes and structural health monitoring.

\section{References}

[1] Iijima, S., Helical microtubules of graphitic carbon. nature, 1991. 354(6348): p. 56.

[2] Eringen, A. C., Nonlocal polar elastic continua. International journal of engineering science, 1972. 10(1): p. 1-16.

[3] Eringen, A. C., On differential equations of nonlocal elasticity and solutions of screw dislocation and surface waves. Journal of applied physics, 1983. 54(9): p. 4703-4710.

[4] Eringen, A. C., Nonlocal continuum field theories. 2002: Springer Science \& Business Media.

[5] Eringen, A. C. and D. G. B. Edelen, On nonlocal elasticity. International Journal of Engineering Science, 1972. 10(3): p. 233-248.

[6] Aydogdu, M., A general nonlocal beam theory: its application to nanobeam bending, buckling and vibration. Physica E: Low-dimensional Systems and Nanostructures, 2009. 41(9): p. 1651-1655.

[7] Reddy, J., Nonlocal theories for bending, buckling and vibration of beams. International Journal of Engineering Science, 2007. 45(2-8): p. 288-307.

[8] Wang, C. M., Y. Y. Zhang, and X. Q. He, Vibration of nonlocal Timoshenko beams. Nanotechnology, 2007. 18(10): p. 105401.

[9] Behera, L. and S. Chakraverty, Free vibration of Euler and Timoshenko nanobeams using boundary characteristic orthogonal polynomials. Applied Nanoscience, 2014. 4(3): p. 347-358.

[10] Wu, L.-Y., et al., Vibrations of nonlocal Timoshenko beams using orthogonal collocation method. Procedia Engineering, 2011. 14: p. 2394-2402.

[11] Eltaher, M., A. E. Alshorbagy, and F. Mahmoud, Vibration analysis of Euler-Bernoulli nanobeams by using finite element method. Applied Mathematical Modelling, 2013. 37(7): p. 4787-4797.

[12] Beni, Y. T., A. Jafaria, and H. Razavi, Size effect on free transverse vibration of cracked nano-beams using couple stress theory. International Journal of EngineeringTransactions B: Applications, 2014. 28(2): p. 296-304.

[13] Hasheminejad, S. M., et al., Free transverse vibrations of cracked nanobeams with surface effects. Thin Solid Films, 2011. 519(8): p. 2477-2482.

[14] Loghmani, M. and M. R. Hairi Yazdi, An analytical method for free vibration of multi cracked and stepped nonlocal nanobeams based on wave approach. Results in Physics, 2018. 11: p. 166-181.

[15] Roostai, H. and M. Haghpanahi, Vibration of nanobeams of different boundary conditions with multiple cracks based on nonlocal elasticity theory. Applied Mathematical Modelling, 2014. 38(3): p. 1159-1169.

[16] Loya, J., et al., Free transverse vibrations of cracked nanobeams using a nonlocal elasticity model. Journal of Applied Physics, 2009. 105(4): p. 044309. 
[17] Sourki, R. and S. Hoseini, Free vibration analysis of sizedependent cracked microbeam based on the modified couple stress theory. Applied Physics A, 2016. 122(4): p. 413.

[18] Sourki, R. and S. Hosseini, Coupling effects of nonlocal and modified couple stress theories incorporating surface energy on analytical transverse vibration of a weakened nanobeam. The European Physical Journal Plus, 2017. 132(4): p. 184.

[19] Bahrami, A., A wave-based computational method for free vibration, wave power transmission and reflection in multicracked nanobeams. Composites Part B: Engineering, 2017. 120: p. 168-181.

[20] Wang, K. and B. Wang, Timoshenko beam model for the vibration analysis of a cracked nanobeam with surface energy. Journal of Vibration and Control, 2015. 21(12): p. 2452-2464.

[21] Torabi, K. and J. Nafar Dastgerdi, An analytical method for free vibration analysis of Timoshenko beam theory applied to cracked nanobeams using a nonlocal elasticity model. Thin Solid Films, 2012. 520(21): p. 6595-6602.

[22] Soltanpour, M., et al., Free transverse vibration analysis of size dependent Timoshenko FG cracked nanobeams resting on elastic medium. Microsystem Technologies, 2017. 23(6): p. 1813-1830.

[23] Civalek, Ö. and B. Akgöz, Free vibration analysis of microtubules as cytoskeleton components: nonlocal EulerBernoulli beam modeling. Scientia Iranica. Transaction B, Mechanical Engineering, 2010. 17(5): p. 367.
[24] Osman, T., et al., Applied and Computational Mathematics Analysis of cracked plates using localized multi-domain differential quadrature method. Vol. 2. 2013. 109-114.

[25] Ragb, O., L. Seddek, and M. Matbuly, Iterative differential quadrature solutions for Bratu problem. Computers \& Mathematics with Applications, 2017. 74(2): p. 249-257.

[26] Zong, Z., K. Y. Lam, and Y. Y. Zhang, A multidomain Differential Quadrature approach to plane elastic problems with material discontinuity. Mathematical and Computer Modelling, 2005. 41(4): p. 539-553.

[27] Civalek, Ö. and Ç. Demir, Bending analysis of microtubules using nonlocal Euler-Bernoulli beam theory. Applied Mathematical Modelling, 2011. 35(5): p. 2053-2067.

[28] Shu, C. and H. Du, Implementation of clamped and simply supported boundary conditions in the GDQ free vibration analysis of beams and plates. International Journal of Solids and Structures, 1997. 34(7): p. 819-835.

[29] Shu, C., Differential Quadrature and Its Application in Engineering. 2000: Springer Science \& Business Media.

[30] Matbuly, M., O. Ragb, and M. Nassar, Natural frequencies of a functionally graded cracked beam using the differential quadrature method. Applied mathematics and computation, 2009. 215(6): p. 2307-2316.

[31] Nassar, M., M. S. Matbuly, and O. Ragb, Vibration analysis of structural elements using differential quadrature method. Journal of Advanced Research, 2013. 4(1): p. 93-102. 